\title{
Gene Therapy Approaches
}

\author{
Ahmed Jumaa Ahmed \\ College of Education -Akre, \\ Department of Biology, \\ University of Dohuk, Duhok, Iraq \\ ahmed.ahmed@uod.ac
}

\author{
Hogir Saadi \\ Ministry of Higher Education \\ Kurdistan Region \\ Iraq \\ dna_proten@yahoo.com
}

\begin{abstract}
Gene therapy can be described broadly as the transfer of genetic material to control a disease, or at least to enhance a patient's clinical status. The transformation of viruses into genetic shuttles is one of the core principles of gene therapy, which will introduce the gene of interest into the target tissue and cells. To do this, safe strategies have been invented, using many viral and non-viral vector delivery. Two major methods have emerged: modification in vivo and modification ex vivo. Non-viral vectors are much a lesser amount of successful compared to other vectors, but because of their low immune responses and their broad therapeutic DNA ability, they have advantages. The addition of viral functions such as receptor-mediated uptake and nuclear translocation of DNA may eventually lead to the development of an artificial virus in order to improve the role of non-viral vectors. In human uses in genetic conditions, tumors and obtained illnesses, gene transfer techniques have been allowed. The ideal delivery vehicle has not been identified, although accessible vector systems are capable of transporting genes in vivo into cells. Therefore, only with great caution can the present viral vectors be used in human beings and further progress in the production of vectors is required. Current progresses in our understanding of gene therapy approaches and their delivery technology, as well as the vectors used to deliver therapeutic genes, are the primary goals of this review. For that reason, a literature search on PubMed and Google Scholar was carried out using different keywords.
\end{abstract}

Keywords-Gene Therapy, Somatic cell, Germ-line cell, DNA, Genetic disease

\section{INTRODUCTION}

Gene therapy is the process or utilization of genetic material such the DNA and RNA [nucleic acids] in therapeutic delivery into human cells in order to treat or prevent diseases. The process of Gene therapies usually comprises the addition of an effective gene into cells to improve cellular dysfunction or to provide a new cellular function. To correct or modify the faulty responsible genes, the technology of gene therapy may be used. In the treatment of combined immunodeficiency syndromes, gene therapy has been particularly successful, demonstrate enduring and impressive therapeutic efficacy [1]. A reasonably huge fragment of genetic material $(>1 \mathrm{~kb})$ is needed in certain cases, including the promoter sequences that enable gene expression, the coding sequences that guide protein production and the signaling sequences that direct the processing of RNA, such as polyadenylation. The second gene therapy class requires modifying the expression in a cell of an endogenous gene [2]. The aim of this review is to discuss current progresses in our consideration of gene therapy approaches, as well as the victors used to deliver therapeutic genes. Using the keywords, a literature search on PubMed and Google Scholar has been implemented. More than 45 papers and documents from the PubMed using the keyword gene therapy and genetic diseases. The other combination of key words regained a number of papers from the database. The most recent and highest cited articles from database search using the combination of keyword germ-line therapy, somatic cell therapy and viral vectors were retrieved. For further study, the results have been assessed, duplications are excluded and related articles in English are used. In the list of references, all the publications used were cited.

\section{GENE THERAPY HISTORY}

Gene therapy was discovered in 1989 at NIH and the successful work was done in 1990. A study by Rosenberg and his colleagues were using a retroviral vector to transfer the neomycin resistance marker gene into tumorinfiltrating lymphocytes collected from five patients with metastatic melanoma in the first clinical trial using gene transfer. These lymphocytes were then expanded in vitro and then re-infused into the patients [3]. Since this first report found that it was feasible and practicable to pass retroviral genes, it has led to several other studies. Indeed, over 900 clinical trials have been licensed worldwide since 1989 [4]. What makes gene therapy effective was the advancement of recombinant DNA technology between 1963 and 1990 [5]. It is recorded that China approved the first gene therapy drugs in 2003 and November 2005 for the treatment of some malignant tumors. In 2005, the European Agency for the Assessment of Pharmaceutical Products (EMEA) submitted the first European application for approval of a gene therapy drug for the treatment of an active brain tumor. The achievements of genome editing can certainly be verified today, despite the ongoing significant challenges in technological implementation. For example, over the past five years, promising treatments have been developed for patients with serious inherited immunodeficiency diseases. To these patients with lifethreatening illnesses, these therapies are visibly helpful. In the USA the death of a patient in 1999 as a consequence of very large dosages of adenoviral vectors delivered systemically was a horrific occurrence that was seen by the 
public as a drawback for gene therapy. German scientists have made major contributions in this area, ranging from basic vector host interaction research to clinical trials. They published on the correction of extreme immunodeficiency in adult patients through genome editing, among other items, in 2006 [5].

\section{GENE THERAPY TYPES}

\section{A. Germ-line Gene Therapy}

This involves inserting corrective genes into cells of the germ line, eggs, or sperm cells, which will then also pass on any genetic modifications to future generations. However, while this method of gene therapy has the potential to prevent inherited diseases, it is highly controversial and very little research is currently being done in this field, both for technological and moral issues $[6,45]$.

\section{B. Somatic Cell Gene Therapy}

It means the insertion of a target gene into targeted cells with the end result of curing the patient, but not the potential children of the patient, since these genes are not transferred to the offspring. In other ways, while some of the genes of the patient can be changed to cure a disease, the risk remains that the children of the patient may be affected by the same illness. This is the type of gene therapy that is practiced in most genetic laboratories [7, 45].

\section{TYPES OF VECTORS}

\section{A. Viral Vectors}

Generally, some viruses perform the action of delivering therapeutic genes into target organ or cells. The main viruses that used as a vector to deliver genes are:

\section{- Retroviruses}

A group of viruses that can generate copies of their genomes with double-stranded DNA. It is possible to incorporate these copies of its genome into host tissue chromosomes. A retrovirus is a human immunodeficiency virus (HIV). One of the retrovirus gene therapy issues is that the integrase enzyme is able to place the virus' genome into any position in the host genome; it inserts the genetic material into a chromosome randomly. If genetic material happens to be placed in the middle of one of the host cell's original genes, it will disrupt this gene (insertional mutagenesis). Uncontrolled cell division (i.e., cancer) may occur if the gene happens to be one regulating cell division. The use of zinc finger nucleases has recently started to address this issue [8]. Other methods are also used to overcome this issue such as, CRISPR Genome Editing, which describes targeted mutagenesis involving a programmable DNA scissor consisting of a protein (Cas9) bound to a short RNA [42]. In this case the RNA, called guide RNA (gRNA), determines the target site in the genome by complementary base paring, while the Cas 9 protein is required for the induction of a double-strand DNA break (DSB) at the binding site. Due to its simplicity, CRISPR quickly became a standard technique in biomedical research $[42,43]$. The high efficiency of CRISPR genome editing has further raised high hopes for the generation of genome-edited plants and farm animals and for novel therapies for inherited human diseases. On the other hand, Zinc-finger nucleases (ZFNs) and transcription activator-like effector nucleases
(TALENs) comprise a powerful class of tools that are redefining the boundaries of biological research. These chimeric nucleases are composed of programmable, sequence-specific DNA-binding modules linked to a nonspecific DNA cleavage domain. ZFNs and TALENs enable a broad range of genetic modifications by inducing DNA double-strand breaks that stimulate error-prone nonhomologous end joining or homology-directed repair at specific genomic locations [44].

\section{- Adenovirus}

Studies have found that some other types of viruses are used to prevent the issue of injecting genes. The genome from double strand DNA viruses that are able to cause pulmonary, gastrointestinal and eye infections. Adenovirus genetic material is not integrated into the genetic material of the host cell [9]. In the nucleus of the host cell, the DNA molecule is left free, and the instructions are transcribed just like every other gene in this extra DNA molecule. Adenovirus can also target a wider range of cells, for instance, lung tissue, than retroviruses. Nevertheless, the patient's immune system is often more likely to be targeted by adenovirus, and large doses of virus needed for treatment also induce an unnecessary inflammatory response [10].

\section{- $\quad$ Adeno - Associated Viruses (AAVs)}

Adeno Associated Viruses (AAVs) are tiny viruses with a genome of single stranded DNA from the family of Parvovirus. It can inject genetic material with almost $100 \%$ certainty. Researchers assume that most individuals bear AAVs that are unable to cause illness and do not cause an immune response. Scientists have demonstrate animal studies using AAV to correct genetic defects [47]. The key disadvantage of AAV is that it is small, in its natural state, bearing only two genes. Hence, its capacity is fairly small. Since the virus inserts its genes directly into the DNA of the host cell, it may produce unintended genetic damage. Researchers have also had difficulty producing significant amounts of the altered virus. Amsterdam Molecular Therapeutics has recently solved the development problem [11].

\section{- Herpes simplex viruses}

A class of viruses of double-stranded DNA that infect a specific type of cell, neurons. The type 1 herpes simplex virus is a widespread human pathogen leading to the development of cold sore syndrome [5]. Particularly in comparison to other viruses, it has a broad genome that allows scientists to inject more than one target gene into a single virus. HSV is an ideal vector since a large variety of tissues can be infected [12]. Researchers have found out that Herpes Simplex Virus 1 antibodies are popular in humans, but problems are somewhat rare due to herpes infection [13].

\section{B. Non-Viral Vectors}

Non-viral vectors have many advantages compared to virus-derived vectors, for example, the safety of infection without immunogenicity, the nearly limitless extent of the transgene, and the opportunity of concurrent insertion $[14,46]$. Generally, non-viral approaches are divided as follow [15].

\section{- $\quad$ Naked Plasmid DNA}


In recent years, a sequence of techniques have been confirmed for naked plasmid DNA-based gene delivery methodologies, such as a naked plasmid DNA [16]. For antiangiogenic therapy, naked DNA has been used where the fetal liver kinase-1 gene was supplied $[17,18]$. Naked DNA is a desirable non-viral vector because of its intrinsic simplicity, and its ease of bacterial development method [19].

\section{- Cationic lipids}

A significant class of compounds appropriate for transporting negatively charged DNA is cationic liposomes. Which are currently a range of commercial cationic lipid-based recombinant reagents, such as Lipofectin, Neophectin and Transfectam. The use of such reagents in vivo, however, is plagued by their inherent toxicity. The charged head groups are typically quaternary amines, saturated or unsaturated alkyl chains or groups of cholesteryl tails [20]. There are various studies on the use of different cationic lipids as vectors of non-viral gene delivery. Between the initial research by Felgner et al in 1987 and their use in the world's first human gene therapy clinical trial by Nabel et al [21]. In recent times, cationic liposomes have been used to deliver siRNA. Up to $80 \%$ of endogenous gene expression in the liver impaired when siRNA complexes and galactosylated liposomes were delivered to mice [22]. It has been found in a recent study that the mechanism behind the toxicity of cationic liposomes is essentially apoptosis induction [23].

\section{- Polymeric gene carriers}

As non-viral vectors for gene transmission, synthetic polycationic polymers have drawn wide attention. A variety of publications and textbook issues have already been written, explaining different mechanisms by which they work, as well as different biochemical and therapeutic aspects of these processes $[24,25]$. Polyethyleneimine is further correct as it has set a gold standard for non-viral gene delivery [24]. Poly-Llysine is the other synthetic polymer which provide promising result in gene delivery. This is one of the first polymers to be investigated due to its peptidic nature for non-viral gene delivery, i.e., it is biodegradable and thus more appropriate for in vivo processing [26-28].

\section{GENE THERAPY IN DisEASES}

\section{A. Gene Therapy for Cystic Fibrosis Lung Disease}

Genetic engineering may be a natural cure for cystic fibrosis: cystic fibrosis (CF) is a recessive condition characterized by loss of functional mutations in the gene of the $\mathrm{CF}$ transmembrane conductance regulator (CFTR), which has a well-characterized gene product; heterozygotes tend to be phenotypically perfectly normal, as predicted; the degree of CFTR expression in affected cells is normally a wellcharacterized gene product [5]. After an enormous amount of research in this field, however, there is little evidence to suggest that a successful approach to gene transfer is imminent for the treatment of CF lung disease. The failure to develop such a cure partly reflects the learning curve with regard to vector technology and the inability to understand the capacity of epithelial airway cells to protect themselves from the outside world against penetration by moieties, like gene therapy vectors.

\section{B. Gene Therapy for Parkinson's Disease}

Parkinson disease is the most commonly known chronic and a progressive neuro-degenerative disease due to the deep degeneration of mid-brain dopamine nigrostriatal neurons associated with extreme motor symptoms. Nevertheless, with numerous etiological variables and pathogenic mechanisms, diverse pathology and a wide variety of central nervous system (CNS) and non-CNS symptoms, PD is much more complex than generally known [29]. With progressive pathology, the efficacy of the medications decreases, leading to incremental patient incapacitation through increased periods of no symptomatic relief and increasing adverse effects such as peak dose dyskinesias. Therefore, effective care appears to reflect a substantial medical need for nigrostriatal-mediated motor impairments, affecting over 4 million people globally [30]. While a range of solutions have been developed to enhance the function of the deteriorating dopaminergic system, it has been difficult to translate this bio-pharmaceutical idea to the clinic because of challenges associated with the continuous and targeted delivery of macromolecules to the central nervous system. Over the past decade, progress made in the field of gene therapy has provided alternatives to several of the delivery restrictions and many aspects of PD freely present it as an ideal clinical indication to target using GT [5].

\section{Gene Therapy for Infectious Diseases}

As a potential cure for a wide variety of infectious diseases that are not amenable to traditional clinical management, gene therapy is being studied [31]. Infectious disease genome editing approaches can be classified into three broad categories: (I) nucleic acid-based gene therapies, (ii) protein strategies such as trans-dominant negative proteins and (iii) immunotherapeutic advances involving genetic vaccines [32]. It is also essential to concurrently use combinations of the above methods to inhibit several stages of the cycle of viral life. The efficacy of gene therapy against infectious agents is the exact consequence of numerous reasons: (I) the specification of the suitable target cell for gene therapy; (ii), the effectiveness of the gene delivery method; (iii) the adequate expression, modulation and maintenance of the product of gene therapy; and (iv) the effectiveness of gene replication inhibition [5].

\section{Gene Therapy for Arthritis}

Rheumatoid arthritis describes as chronic autoimmune disorders characterized by synovial and intra-articular inflammation that consequences in the degradation of cartilage and bone tissue [33]. Biological agents that inhibit proinflammatory cytokine actions have currently seen efficacy as anti-arthritic drugs, however involve regular administration. As an alternative strategy to target, more effective and sustained delivery of inflammatory cytokine inhibitors as well as other therapeutic agents, gene transfer approaches are therefore being established [34]. Biological agents modulating the proinflammatory activities of TNF and IL1 have recently been shown to be effective as potential anti-arthritic drugs [35]. There are some different methods that can be used for arthritis treatment [36]. Genes may be administered locally by intra-articular injection at 
the site of disease pathology, such as the joint. Restorative genes may alternatively be delivered using particular types of circulating cells, such as T cells [37, 38]. Even though these cell types result in a more systemic distribution of therapeutic proteins, local therapy after systemic injection may also be possible for some immune regulatory cells to be able to access their home sites of inflammation. Through supplying the gene to tissues such as the muscle or liver, the levels of circulating therapeutic proteins may also be increased [39].

\section{ETHICAL CONSIDERATION IN GENE THERAPY}

Gene therapy involves manipulation or editing of humans body or organs or organ systems through basic instructions passed through cells/genetic materials, therefore the approach attracts ethical consideration a lot. In the field of genetics, the prospect of genetically altering germ-line has long been the topic of intense debate. In order to determine the risks of the operation and the moral consequences involved, bioethics is often present when new technologies are developed. Genome therapy for somatic cells is accepted by a large part of the scientific community, especially in cases of serious disorders such as cystic fibrosis [40, 41]. To track their growth and wellbeing, follow-up on children born after GGT would be important [48]. There is a controversy about how long it is appropriate to track children, with views varying from a few years or even decades, or even centuries to generations $[6,32]$.

\section{CONCLUSION}

In conclusion, the degree to which treatment can be used depends on the interpretation of the process. It would seem that some genetic disorders that were not accessible with previous techniques would be accessible with gene-based therapy. Particularly, the potential for autosomal treatment in both of these approaches, dominant conditions are a function. Furthermore, it has prompted some suggest that gene therapy might potentially make a genetic defect permanent and conclusive correction of the germ-line possible. For the effective and stable distribution of therapeutic DNA, a variety of methods have been and are being invented. However, the growing variety also suggests that there is no simple ideal delivery method to date. In this area of molecular biomedicine, the insights and hopes associated with gene therapy support science.

\section{REFERENCES}

[1] M. Cavazzana-Calvo et al., "Gene therapy of human severe combined immunodeficiency (SCID)-X1 disease," (in eng), Science, vol. 288 no. 5466, pp. 669-72, Apr 28 2000, doi: 10.1126/science.288.5466.669.

[2] M. Cavazzana-Calvo, S. Hacein-Bey, F. Yates, J. P. de Villartay, F. Le Deist, and A. Fischer, "Gene therapy of severe combined immunodeficiencies," (in eng), J Gene Med, vol. 3, no. 3, pp. 201-6, May-Jun 2001, doi: 10.1002/1521-2254(200105/06)3:3<201::aidjgm195>3.0.co;2-z.

[3] S. A. Rosenberg et al., "Gene transfer into humans--immunotherapy of patients with advanced melanoma, using tumor-infiltrating lymphocytes modified by retroviral gene transduction," (in eng), $\mathrm{N}$ Engl J Med, vol. 323, no. 9, pp. 570-8, Aug 30 1990, doi: 10.1056/nejm199008303230904.

[4] M. L. Edelstein, M. R. Abedi, J. Wixon, and R. M. Edelstein, "Gene therapy clinical trials worldwide 1989-2004-an overview," (in eng), J Gene Med, vol. 6, no. 6, pp. 597-602, Jun 2004, doi: 10.1002/jgm.619.
[5] M. Mishra, "A review on gene therapy: History, vectors, technologies and application," WORLD JOURNAL OF PHARMACY AND PHARMACEUTICAL SCIENCES, vol. 5, 01/26 2018, doi: 10.20959/wjpps201610-7891.

[6] D. P. Wolf, P. A. Mitalipov, and S. M. Mitalipov, "Principles of and strategies for germ-line gene therapy," (in eng), Nat Med, vol. 25, no. 6, pp. 890-897, Jun 2019, doi: 10.1038/s41591-019-0473-8.

[7] A. Bank, "Human somatic cell gene therapy," BioEssays, https://doi.org/10.1002/bies.950181210 vol. 18, no. 12, pp. 999-1007, 1996/12/01 1996, doi: https://doi.org/10.1002/bies.950181210.

[8] S. Durai, M. Mani, K. Kandavelou, J. Wu, M. H. Porteus, and S. Chandrasegaran, "Zinc finger nucleases: custom-designed molecular scissors for genome engineering of plant and mammalian cells," (in eng), Nucleic Acids Res, vol. 33, no. 18, pp. 5978-90, 2005, doi: 10.1093/nar/gki912.

[9] Z. Peng, "Current status of gendicine in China: recombinant human Ad-p53 agent for treatment of cancers," (in eng), Hum Gene Ther, vol. 16, no. 9, pp. 1016-27, Sep 2005, doi: 10.1089/hum.2005.16.1016.

[10] B. Sibbald, "Death but one unintended consequence of gene-therapy trial," in Cmaj, vol. 164, no. 11), 2001, p. 1612.

[11] J. L. Santiago-Ortiz and D. V. Schaffer, "Adeno-associated virus (AAV) vectors in cancer gene therapy," (in eng), J Control Release, vol. 240, pp. 287-301, Oct 28 2016, doi: 10.1016/j.jconrel.2016.01.001

[12] W. Walther and U. Stein, "Viral vectors for gene transfer: a review of their use in the treatment of human diseases," (in eng), Drugs, vol. 60, no. 2, pp. 249-71, Aug 2000, doi: 10.2165/00003495-20006002000002.

[13] M. Hao, C. Huang, and N. Xia, "[The Advances of Oncolytic Herpes Simplex Virus in Cancer Therapy]," (in chi), Bing Du Xue Bao, vol. 32, no. 4, pp. 516-22, Jul 2016.

[14] R. Gardlík, R. Pálffy, J. Hodosy, J. Lukács, J. Turna, and P. Celec, "Vectors and delivery systems in gene therapy," (in eng), Med Sci Monit, vol. 11, no. 4, pp. Ra110-21, Apr 2005.

[15] T. G. Park, J. H. Jeong, and S. W. Kim, "Current status of polymeric gene delivery systems," (in eng), Adv Drug Deliv Rev, vol. 58, no. 4, pp. 467-86, Jul 7 2006, doi: 10.1016/j.addr.2006.03.007.

[16] H. Yoshino, K. Hashizume, and E. Kobayashi, "Naked plasmid DNA transfer to the porcine liver using rapid injection with large volume," (in eng), Gene Ther, vol. 13, no. 24, pp. 1696-702, Dec 2006, doi: 10.1038/sj.gt.3302833.

[17] H. Yazawa et al., "Hydrodynamics-based gene delivery of naked DNA encoding fetal liver kinase-1 gene effectively suppresses the growth of pre-existing tumors," (in eng), Cancer Gene Ther, vol. 13, no. 11, pp. 993-1001, Nov 2006, doi: 10.1038/sj.cgt.7700970.

[18] M. Cemazar, M. Golzio, G. Sersa, M. P. Rols, and J. Teissié, "Electrically-assisted nucleic acids delivery to tissues in vivo: where do we stand?," (in eng), Curr Pharm Des, vol. 12, no. 29, pp. 3817-25, 2006, doi: 10.2174/138161206778559740.

[19] J. A. Wolff and V. Budker, "The mechanism of naked DNA uptake and expression," (in eng), Adv Genet, vol. 54, pp. 3-20, 2005, doi: 10.1016/s0065-2660(05)54001-X.

[20] M. Jafari, M. Soltani, S. Naahidi, D. N. Karunaratne, and P. Chen, "Nonviral approach for targeted nucleic acid delivery," (in eng), Curr Med Chem, vol. 19, no. 2, pp. 197-208, 2012, doi: 10.2174/092986712803414141.

[21] E. G. Nabel and G. J. Nabel, "Direct gene transfer: basic studies and human therapies," (in eng), Thromb Haemost, vol. 70, no. 1, pp. 2023, Jul 11993.

[22] A. Sato, M. Takagi, A. Shimamoto, S. Kawakami, and M. Hashida, "Small interfering RNA delivery to the liver by intravenous administration of galactosylated cationic liposomes in mice," (in eng), Biomaterials, vol. 28, no. 7, pp. 1434-42, Mar 2007, doi: 10.1016/j.biomaterials.2006.11.010.

[23] L. T. Nguyen, K. Atobe, J. M. Barichello, T. Ishida, and H. Kiwada, "Complex formation with plasmid DNA increases the cytotoxicity of cationic liposomes," (in eng), Biol Pharm Bull, vol. 30, no. 4, pp. 751-7, Apr 2007, doi: 10.1248/bpb.30.751.

[24] A. Rolland, "Gene medicines: the end of the beginning?," (in eng), Adv Drug Deliv Rev, vol. 57, no. 5, pp. 669-73, Apr 5 2005, doi: 10.1016/j.addr.2005.01.002. 
[25] T. Azzam and A. J. Domb, "Current developments in gene transfection agents," (in eng), Curr Drug Deliv, vol. 1, no. 2, pp. 165 93, Apr 2004, doi: 10.2174/1567201043479902.

[26] M. A. Wolfert et al., "Polyelectrolyte vectors for gene delivery: influence of cationic polymer on biophysical properties of complexes formed with DNA," (in eng), Bioconjug Chem, vol. 10, no. 6, pp. 993-1004, Nov-Dec 1999, doi: 10.1021/bc990025r.

[27] J. M. Benns, J. S. Choi, R. I. Mahato, J. S. Park, and S. W. Kim, "pHsensitive cationic polymer gene delivery vehicle: N-Ac-poly(Lhistidine)-graft-poly(L-lysine) comb shaped polymer," (in eng), Bioconjug Chem, vol. 11, no. 5, pp. 637-45, Sep-Oct 2000, doi: 10.1021/bc0000177.

[28] I. Fajac et al., "Histidylated polylysine as a synthetic vector for gene transfer into immortalized cystic fibrosis airway surface and airway gland serous cells," (in eng), J Gene Med, vol. 2, no. 5, pp. 368-78, Sep-Oct 2000, doi: 10.1002/1521-2254(200009/10)2:5<368::aidjgm118>3.0.co;2-f.

[29] L. V. Kalia and A. E. Lang, "Parkinson's disease," (in eng), Lancet, vol. 386, no. 9996, pp. 896-912, Aug 29 2015, doi: 10.1016/s01406736(14)61393-3.

[30] C. W. Olanow, M. B. Stern, and K. Sethi, "The scientific and clinical basis for the treatment of Parkinson disease (2009)," (in eng), Neurology, vol. 72, no. 21 Suppl 4, pp. S1-136, May 26 2009, doi: 10.1212/WNL.0b013e3181a1d44c.

[31] A. M. Keeler, M. K. ElMallah, and T. R. Flotte, "Gene Therapy 2017: Progress and Future Directions," (in eng), Clin Transl Sci, vol. 10, no. 4, pp. 242-248, Jul 2017, doi: 10.1111/cts. 12466.

[32] I. Gabel and J. Moreno, "Genome Editing, Ethics, and Politics," (in eng), AMA J Ethics, vol. 21, no. 12, pp. E1105-1110, Dec 1 2019, doi: 10.1001/amajethics.2019.1105.

[33] J. S. Smolen, D. Aletaha, and I. B. McInnes, "Rheumatoid arthritis," (in eng), Lancet, vol. 388, no. 10055, pp. 2023-2038, Oct 222016 , doi: 10.1016/s0140-6736(16)30173-8.

[34] S. Liu and K. Maeyama, "Gene Therapy for Rheumatoid Arthritis," (in eng), Crit Rev Immunol, vol. 36, no. 2, pp. 149-161, 2016, doi: 10.1615/CritRevImmunol.2016017062.

[35] Y. Jiang et al., "A multicenter, double-blind, dose-ranging, randomized, placebo-controlled study of recombinant human interleukin-1 receptor antagonist in patients with rheumatoid arthritis: radiologic progression and correlation of Genant and Larsen scores," (in eng), Arthritis Rheum, vol. 43, no. 5, pp. 1001-9, May 2000, doi: 10.1002/1529-0131(200005)43:5<1001::aid-anr7>3.0.co;2-p.
[36] J. N. Gouze et al., "Gene therapy for rheumatoid arthritis," (in eng), Hand Surg, vol. 6, no. 2, pp. 211-9, Dec 2001, doi: 10.1142/s0218810401000709.

[37] A. Nakajima et al., "Antigen-specific T cell-mediated gene therapy in collagen-induced arthritis," (in eng), J Clin Invest, vol. 107, no. 10, pp. 1293-301, May 2001, doi: 10.1172/jci12037.

[38] S. H. Kim, S. Kim, T. J. Oligino, and P. D. Robbins, "Effective treatment of established mouse collagen-induced arthritis by systemic administration of dendritic cells genetically modified to express FasL," (in eng), Mol Ther, vol. 6, no. 5, pp. 584-90, Nov 2002.

[39] N. Saidenberg-Kermanac'h et al., "Efficacy of interleukin-10 gene electrotransfer into skeletal muscle in mice with collagen-induced arthritis," (in eng), J Gene Med, vol. 5, no. 2, pp. 164-71, Feb 2003, doi: 10.1002/jgm.321.

[40] G. A. R. Gonçalves and R. M. A. Paiva, "Gene therapy: advances, challenges and perspectives," (in eng por), Einstein (Sao Paulo), vol. 15, no. 3, pp. 369-375, Jul-Sep 2017, doi: 10.1590/s1679$45082017 \mathrm{rb} 4024$.

[41] A. V. Levin, "Ethical considerations in gene therapy," (in eng), Ophthalmic Genet, vol. 37, no. 3, pp. 249-51, Sep 2016, doi: 10.3109/13816810.2015.1121501

[42] C. Brakebusch, "CRISPR Genome Editing: How to Make a Fantastic Method Even Better," Cells, vol. 10, no. 2, 2021, doi: 10.3390/cells10020408.

[43] P. Sledzinski, M. Nowaczyk, and M. Olejniczak, "Computational Tools and Resources Supporting CRISPR-Cas Experiments," (in eng), Cells, vol. 9, no. 5, May 22 2020, doi: 10.3390/cells9051288.

[44] T. Gaj, C. A. Gersbach, and C. F. Barbas, 3rd, "ZFN, TALEN, and CRISPR/Cas-based methods for genome engineering," (in eng), Trends Biotechnol, vol. 31, no. 7, pp. 397-405, Jul 2013, doi: 10.1016/j.tibtech.2013.04.004

[45] S. Patil, I. A. Al-Zoubi, P. H. Raghuram, N. Misra, N. Yadav, and M. Alam, "Gene therapy: A comprehensive review," International Medical Journal, vol. 25, pp. 361-364, 12/01 2018.

[46] S. Ginn and I. Alexander, "Gene Therapy," 2016, pp. 1-19.

[47] M. Collins and A. Thrasher, "Gene therapy: Progress and predictions," Proceedings. Biological sciences / The Royal Society, vol. 282, 12/24 2015, doi: 10.1098/rspb.2014.3003.

[48] I. Gabel and J. Moreno, "Genome Editing, Ethics, and Politics," (in eng), AMA J Ethics, vol. 21, no. 12, pp. E1105-1110, Dec 1 2019, doi: 10.1001/amajethics.2019.1105. 